\title{
GROUP AND INDIVIDUAL MMPLICIT THEORIES OF PERSONALITY: AN APPLICATION OF THREE-MODE PRINCIPAL CONPONENT ANALYSIS
}

\author{
WILLEM A. VAN DER KLOOT \\ and \\ PIFTER M. KROONENBERG \\ Rijksuniversiteit Leiden
}

\begin{abstract}
Data produced by 60 subjects rating 32 artificial persons on 11 personaiity trait scales were analyzed by threemode principal component analysis. The subjects shared a circular configuration of stimul ard seales. Indiriduais tended to depart from this pattem in extremity of bugement rather than configuration, suggesting the operation of a response style. The advantages of including "artificial subjects" and of partitioning the residuai sums of squares are discussed.
\end{abstract}

The notion that people use naive, common sense, or impticit theories of personality (ITPS) when they form impressions of another person's personality was introduced in 1954 when Bruner and Tagiuri proposed a cognitive process approach to the study of person perception. In its original meaning, a person's ITP is a set of perceived or expected relations among personality traits: these perceptions and expectations may vary from person to person. Since then. numerous studies have been conducted on various aspects of the concept of the ITP. These studies were reviewed by Schneider (1073) who distinguished between two research traditions in this area: one dealing with the judgment processes of a perceiver (e.y., Hays, 1958; Wishner, 1960; Rosenberg, Nelson, and Vivekananthan, 1968: Zanna and Hamilton, 1972; Bryson, 1974; Van der Kloot, 1975), the other with individual differences in person perception (a.g., Jones, 1954; Pedersen, 1965: Messick and Kogan, 1966; Walters and Jackson, 1966; Lay and Jackson, 1969; Wiggins, Hoffman and Taber, 1969; Sherman, 1972; Van der Kloot, 1975; Wiggins and Blackbum. 1976; Rosenberg, 1977; Kim and Rosenberg, 1980).

Although studies on the ITP vary with regard to design, tasks, subjects, and methods of analysis, their results are strikingly similar. It is frequently found that the ITP can be represented in a two-dimensional configuration whose dimensions are evaluation and cominance (or have similar labels). Moreorer; it has been shown

Requests for reprints should be sent to the first anthor. Psychological Institute, Hooigracht $15,2312 \mathrm{KN}$ Leiden, The Nctherlands. 
Whem A. Van der Kloot and Pieter M. Kroonenberg

that a large number of personality descriptors can be summarized by means of a circumplex (Lorr and McNair, 1963; Becker and Krug, 1964; Rirn, 1965; Benjamin, 1974; Wiggins, 1979; see also Wiggins ot al, 1981). The structures found in those stucies are Guite similar to the circumplex model proposed by leary and his associates (Freednan et al, 1951; Leary, 1957). Personality traits and interpersonal behaviors are described in terms of a love-hate and a dominance-submission cimension.

A similar ITP was cbtained by conibining five ITP configura. tions found in studies by Van der Kloot (1975). The combined configuration represented eight personality trait asjectives in two dimengions (evaluation and dominance-submission). The eight traits were lying on a circular contouw. Yan der Kloot and Van den Boogaard (1978) used this configuration to design an impression formation experiment in which 31 hypothetical stimulus persons (11 described by single trait names; 20 described by pairs of traits) were judged on 11 rating scales by 60 subjects. These data were analyzed by means of a carionical discriminant analysis (CADA; cf. Van de Geer, 1971, p. 184) which yielded two dimensions that optimally discriminated between the stimulus persons. Both the loadings of the rating scales and the projections of the stimuli on the two dimensions confimed the cireumplex nature of the traits that were used. Since this CADA yielded configurations that are aggregated across subjects, these results do not necessarily reflect the ITP of each individual. In view of the evidence that there exist indvidual differences in ITPs (e.g. Wiggins and Blackburn, 1976; Kim and Rosenberg, 1980) the Van der Kloot and Van den Boogaard data were re-analyzed in order to explore the extent and nature of individual differences in those data. The present paper describes this re-analysis. It shows how three-mode principal component analysis can be used in such an investigation both at the aggregate and at the individual level. In this way it yields insight in judgment processes and individual differences at the same time.

\section{Models of analysis}

Our data can be arranged in a three-dimensional block with the index $i$ (referring to the stimuli) along the vertical axis, the index $j$ (referring to the scales) along the horizontal axis, and the index $k$ (referring to the subjects) along the 'depth' axis.

The general threemode principal component model the Tuck- 
ers model) can be formulated as the factorization of the threenode data matrix $Z=\left\{z_{i j h}\right\}$ such that

$$
z_{i, k}=\sum_{p=1}^{3} \sum_{q=1}^{t} \sum_{\gamma^{r}=1}^{u} a_{i p} h_{i q} \epsilon_{k n} c_{w q r}
$$

for $i=1, \ldots, l ; j=1, \ldots, m ; k=1, \ldots, n$. The coefficients $g_{p,}$ $h_{j q}$, and $\epsilon_{k}$ are the elements of the component matrices $\mathrm{G}(K \times s)$, $\mathbf{H}(m \times t)$, and $\mathbf{E}(n \times u)$ respectively; $l, m, n$ are the rumber of variables, and $s, t, u$ are the number of components of the first, second and third mode respectively. We will always assume that $G$, $\mathrm{H}$ and $\mathrm{E}$ are columnwise orthonormal matrices with the rumber of rows larger than or equal to the number of columns. The $c_{p q}$ are the elements of the so-called three-mode core matrix $\mathrm{C}(s \times t \times u)$. The core matrix describes how the latent variables for components) of the different modes are related.

In practice, the data block $Z$ is not decomposed into all its components as one usually is only interested in the first few of them. Therefore one seeks an approximate decomposition $\hat{Z}$ via $a$ least squared loss function. The algorithm to solve the minimization problem is implemented in the program TUCKALS3. Details about the existence of a minimum and the algorithm can be found in Kroonenberg and De Leeuw (1080).

An important restriction of the general Tuckers model can be obtained by equating the component matrix $\mathbf{E}$ with the identity matrix. We will refer to this model as the Tucker2 model, which is implemented in the program TUCKALS2. It can be written as

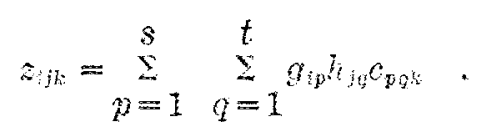

An equivalent matrix notation is

$$
Z_{k}=G_{k} \mathrm{C}_{k} \mathrm{H}^{\prime} \quad\left(k=1, \ldots, n_{i}\right)
$$

where $Z_{k}:(l \times m)$ and $C_{k}(s \times t)$ are two-mode matrices for the $k$ th 
Willem A. Van der Kloot and Pieter N. Kroonenberg

subject; they will be referred to as 'Exontal planes' or "core planes." The Tucker2 model only specifies principal components for the $l$ stimuli and $m$ scales but not for the $n$ subjects. The relationships between the components of the stimuli and the scales can be investigated for each subject separately as well as for all subjects together.

One important advantage of the methods used here over the standard procedures outined by Tucker (1966, p. 297ff) is that the estimates of the parameters are least scuared ones, wher than estimates with ill-defined properties. Another important advantage of the use of loss functions is that it becomes possible to look at residuals. A third advantage is that there exist direct reiationships between the eigenvalues of the configurations and the size of the elements in the core matrix (see e.g. formula [8]).

It can be shown that both for the Tucker2 and Tucker3 model the following equation is true:

$$
\begin{aligned}
& \sum_{i=1}^{l} \sum_{j=1}^{m} \sum_{i=1}^{n} z^{2}{ }_{i j k}= \\
& \sum_{i=1}^{l} \sum_{i=1}^{m} \sum_{k=1}^{n} \hat{z}_{i j k}^{s_{i}}+\sum_{i=1}^{l} \sum_{i=1}^{m} \sum_{k=1}^{n}\left(z_{i j k}-\hat{z}_{i, k}\right)^{2},
\end{aligned}
$$

or

$$
S S(D A T A)=S S(F I T)+\text { SS(RESIDUAL) }
$$

where the $\hat{z}_{i j k}$ 's are the data reconstructed from the estimated parameters of the model. It also holds that for each element $f$ of each mode

$$
\mathrm{SS}\left(\mathrm{DATA}_{f}\right)=\mathrm{SS}\left(\mathrm{FIT}_{f}\right)+\mathrm{SS}\left(\mathrm{RESIDUAL}_{f}\right)
$$

By comparing the fitted sum of squares and the residual sum of squares of, say, the hth subject one can gauge the correspondence of the kth subjects configuration with the overall configuration. 
Note that the contribution of a particular subject to the overal solution can be judged from the absolute size of his SS(FIT).

\section{METHOD}

Stimulus material

The stimulus material used by Van der Kloot and Van den Boogaard consisted of eleven personality trait adjectives: Likeable. cooperative, intelligent, industrious, dominant, aggressive, mieliable, pessimistic, passive, submissive, and modest. Earlier research had shown that these stimuli lie on a circle in the order in which they are presented above. These stimuli were used in two experimental tasks.

In the first task, subjects were presented with descriptions of 11 stimulus persons. Each stimulus person was described by one of the adjectives (for instance: somebody is aroressive). In the second task the subjects had to rate 20 stimulus persons, each described by combinations of two adjectives (see Table 1).

Table 1

Combinations of Adjectives Usecin in Second Experimenta: Tasi

Likeable-cooperative
Conperative-dominant
Intelligent-cooperative
Intellyent-dominant
Intelligent-pessimistic
Dominant-aggressive
Dominant-pessimistic
Dominart-submissive
Aggressive-umreliable
Inrelieble-cooperative

(LI-CO)

(CO-DO)

$(\mathrm{IN}-\mathrm{CO})$

(IN-DO)

(IN-PE)

(DO-AG)

(DO-PE)

(DO-SU)

$(\mathrm{AG}=\mathrm{UN})$

(UN-CO)
Unreliable-intelligent

Unreliable-pessinistic

Pessinistic-aggressire

Pessimistic-passive

Passive-intelligent

Passive-donirant

Passive-aggressive

Passive-unreliable

Passive-submissive

Submissive-pessinistic
$(\mathrm{UN}-\mathrm{IN})$

(UN-PE)

(PE-AG)

(PE-PA)

(PA-IN)

(PA-DO)

(PA-AG)

$(P A-T N)$

(PA-SU)

(SU-PE)

The descriptions of the stimulus persone were presented in two booklets, each preceded by an instruction page. The descriptions were printed on top of each page, and were followed by eleven 10 . point rating scales. These rating scales were labelled with the eleven personality traits mentioned above, including the adjective (or adjectives) used in the description of the stimulus person. The rating scales ranged from 1 to 10 , with end points denoted by "extremely not . .." and "extremely ..." (e.g., "extremely not cooperative" and "extremely cooperative"). The order of the scales on each page of the booklets was randomized, and four different 
Willem $A_{n}$ Van der Kloot and Pieter M. Kroonenberg

random orders of pages were used when the booklets were constructed. The orcers of the two traits in a description used in the second task were determined by chance, but the same order was employed in all presentations. After completing the booklets the subjects were presented a few open-ended questions about their judgment process.

\section{Subiecte}

The subjects participating in this experiment were randomy sampled resicients of a student housing project at the Technical University of Deff, The Netherlands. The sctual experiment was run during two group sessions with respectively 34 and 26 subjects. The whole procedure took about one and one-half hours, for which Dfl. 6.50 (approximately $\$ 3$ ) was paid.

\section{RESULTS}

In the two tasks the subjects rated a total of 31 stimulus persons on 11 criterion variables. These data were analyzed by means of three-mode princival component analysis asing the TUCKALS2 and TUCKALS3 programs. One subject was exchded from the analysis because be or she had too many missing data. For five other subjects who each had one missing score, an estimated rating was substituted. In some analyses, the data set was extended with six artificial 'subjects' in order to improve the interpretability of the solutions. The first, on average-subject (Al), consisted of the mean ratings of the stimuli averaged over the 59 real subjects. The second, or dominancsubject (A2), was construeted as if he judged the stimuli only with respect to their apparent dominance. The third, or evaluation-subrect (A3), was constructed as if the subject only juaged the evaluative content of the stimul. The fourth, or random-subiect (A4), consisted of uniform random error superimposed on the overall seale means. The data of the fifth, or uniform-scorer (A5), were ecual to the grand mean, i.e. the average over stimuli, scales and subjects. The ratings of the sixth, or extreme-scorer (A6), consisted of either 2 or 9 scores. His scores were equal to 9 when the ratings of the average subiect after being centered) were larger than 0 . His scores were 2 when the average-subjects' double-centered ratinge were smaller than 0 . We will use the artificial subjects as some other authors have used 
Willem $A$. Van der Kloot and Pieter $M$. Kroonenberg 'conceptual individuals' or 'idealized individuals' (e.g. Tucker and Messick, 1963; Cliff, 1968; Tucker, 1972). The advantage of onr artificial subjects is that they were created on the basis of possible scoring behaviors of individuals. When included in the analysis they provide $a$ priori and sample-independent information about the subject space which is not the case with 'conceptual individuals.'

Several TUCKALS2 (T2) and TUCKALS3 (T3) analyses were performed, varying with regard to (a) the number of stbjects $(59$ or 65 ), and (b) the dimensionality of the stimulus and scale spaces. In general, we will present the results for the 59 real subjects in two dimensions when discussing these spaces. We will discuss the artificial subjects only in connection with the subject space of the T3 analysis (this analysis is indicated by T3-65).

The data were 'double-centered' per subject matrix. i.e,

$$
z_{i j k}^{*}=z_{i j k}-\bar{z}_{i j k}-\bar{z}_{i, k}+\bar{z}_{. . .5}
$$

This centering removes some sources of unwanted variance (cf. Cronbach, 1955). Thus the different subjects were made identical with respect to scale and stimulus means, leaving the configurational aspect of the ITP's (i.e., the stimulus $\times$ scale interactions) as the data to be analyzed.

\section{Scales}

The two-dimensional T2 configuration of the scales explained 52 percent of the total sum of squares. Since a third dimension reduced the residual sum of squares by only 4 percent, we found the two-dimensional solution quite satisfactory, especially becase the first two dimensions of the threedimensional solution were virtually identical to those of the two-dimensional configuration. Therefore, we will only discuss the two-dimensional solutions. The configuration of the rating seales is pictured in Pigure 1 after a slight orthonormal rotation (see below) of the principal components. Each dimension explains an almost egual amount of variation: respectively 26.7 and 25.6 percent. This indicates that they are of equal importance for the group as a whole. The shape of the T2 configuration is roughly circular, and the horizontal and vertical dimensions can be interpreted as a dominance-submission and an evaluation dimension. 
Willen A. Van der Kioot and Pieter M. Kroonenberg

It is possible to compare the SS(FIT)/SS(RESIDUAL) ratio of the separate scales with each other. It appears that intelligent has the smallest ratio, and therefore fits less well in the total structure than the other scales. This probably means that there are relatively large inter-subject aifferences with regard to the position of intelligent that cannot be accommodated in the model.

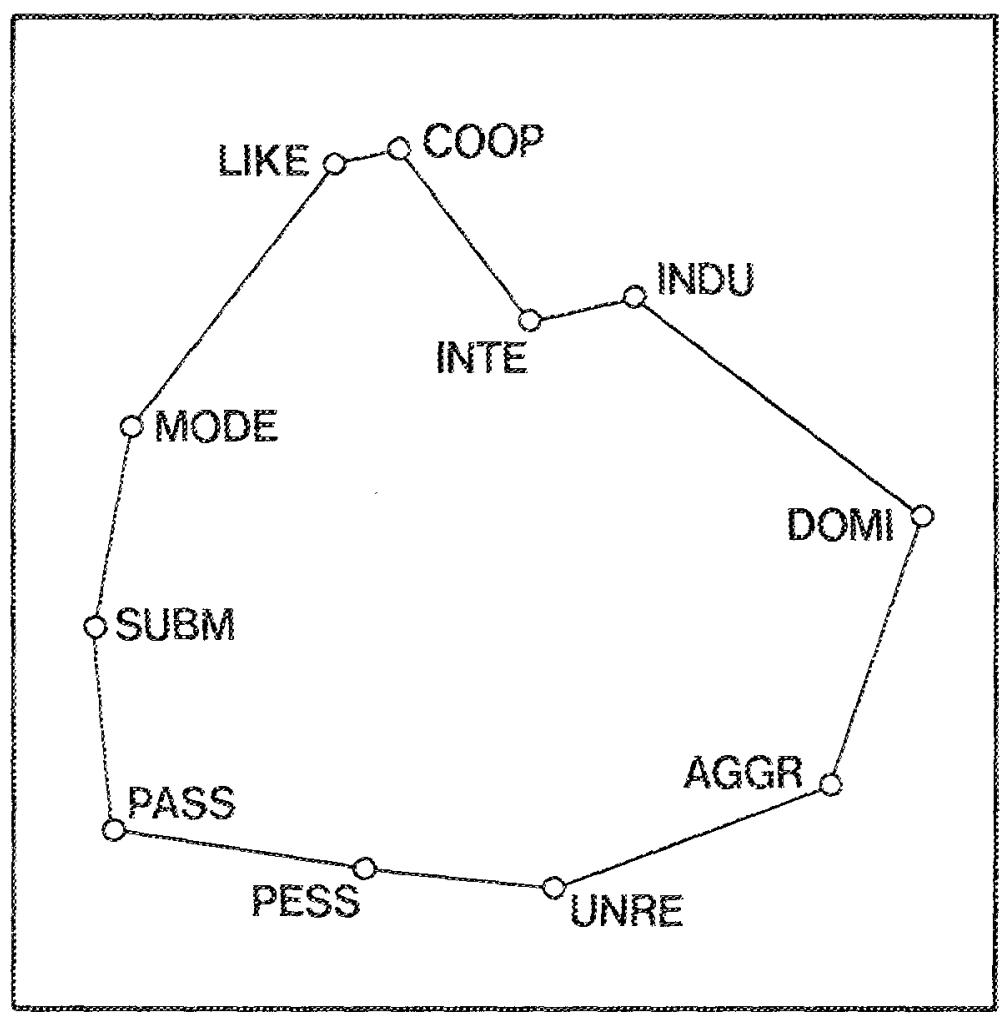

Fig. 1. Scale Configuration of TUCKALS2 Analysis.

\section{Stimuli}

The T2 configuration of the stimul is represented in Figure 2 . The 11 stimuli consisting of single adjectives lie on a polygon which is more or less the same as that of the scales in Figure 1 , with the exception of intelligent and industrious which have switched places. Notwithstanding this difference, one may conclude that the stimulus space and the scale space are virtually identical; these spaces and their respective dimensions seem to have the same 
Willem A. Van der Kloot and Pieter M. Kroonenberg cognitive structure. Moreover, the two dimensions of the stimulus space also account for an almost equal proportion of the sum of squares (resp. 26.6 and 25.6 percent). Therefore, Figure $I$ and Figure 2 may directly be superimposed without further standardization of the projections, and we will refer to both the scale and the stimulus space as the (personality) trait space (or ITP).

The SS(FIT)/SS(RESLDUAL) ratios of the separate stimuli show that the stimuli intelligent-pessimistic, dominant-submissive, umreliable-cooperative, and passive-dominant have much smaller contributions to the SS(FIT) than the other stimul.

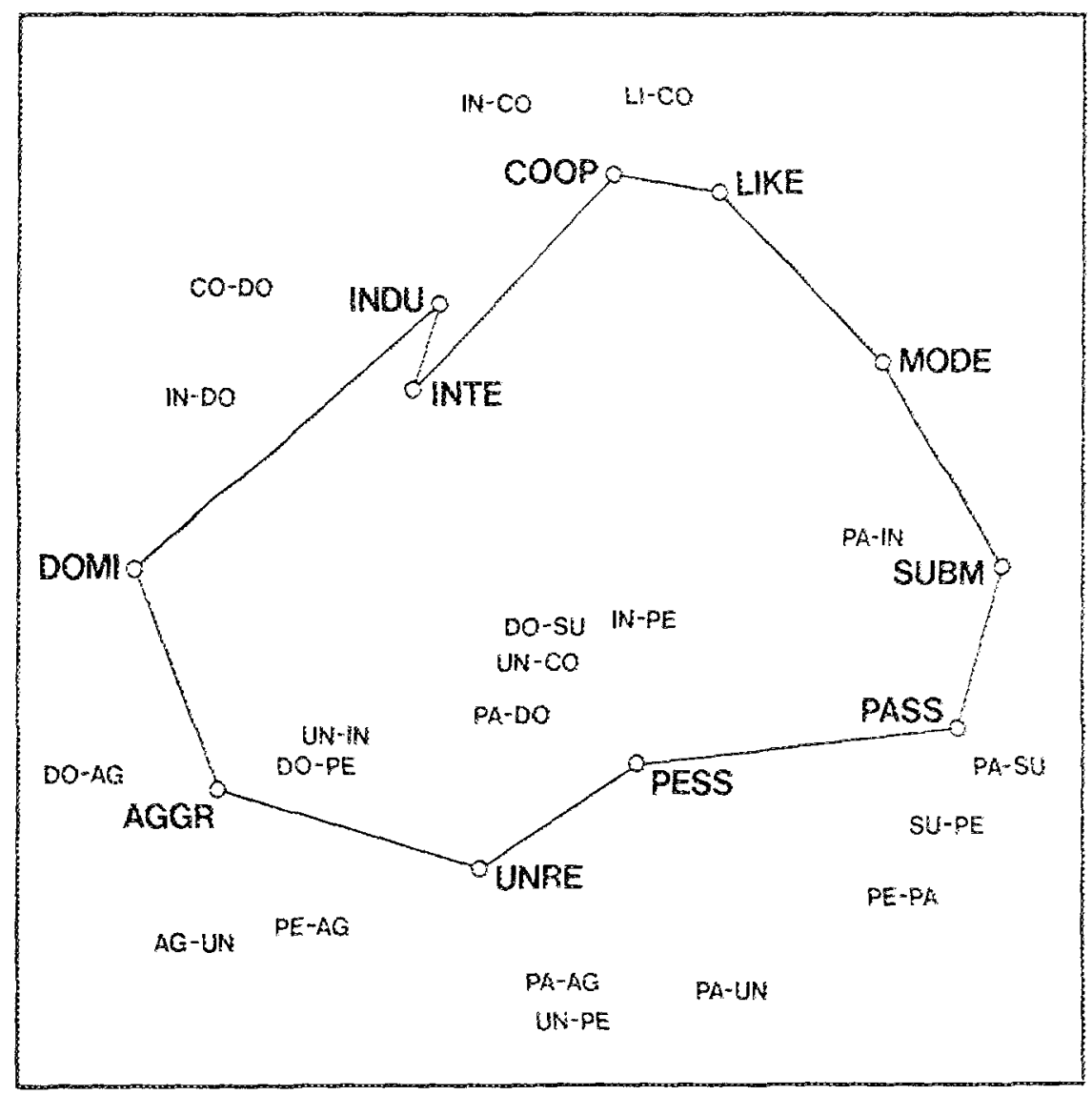

Fig. 2. Stimulus Configuration of TUCKALS2 Analysis.

So far, the T2 results imply that different subjects to a greater or lesser extert share the same underlying ITP. The Tucker2 
Whiem A. Van der Kioot and Pieter M. Kroonenberg

model, however, allows for individual variations consisting of linear transformations of these spaces. The subject is allowed to rotate (orthogonally or obliquely) the dimensions of these configurations, and is also free to stretch or shrink these dimensions. The parameters describing these individual transfomations are collected in a $2 \times 2 \times 59$ extended core matrix which can be sliced into 59 "frontal planes." The interpretability of the frontal planes is improved if the core matrix is rotated so that it is as diagonal as possible in each of its frontal planes. If perfect diagonality is achieved the first (second) component of the stimuli is completely linked to the first (second) component of the seales, and a common interpretation can be given to the components. Such a rotational procedure is included in the TUCKALS2 program (for details see Kroonenberg and De Leenw, 1977), and was applied to our T2 solution. The improvements were very slight, mainly because the core matrix was already almost diagonal. This supports the above assertion that the dimensions of the stimulus and scale spaces have the same mearing.

Before proceeding to a detailed discussion of the subject space it should be mentioned that the Ty configuration of the scales and the stimuil were virtually jentical to those of $\mathrm{T} 2$, both for the 59 and 65 subject solutions. The absolute difference between the coordinates of the $\mathrm{T} 2$ and the $\mathrm{T} 3$ configurations ranged from zero to .0075 , the average absolute difference being .0020 .

Subjects: overall analysis

$T g$ subject space. The eigenvalues of the two components of the subject space from the T3-65 analysis were respectively 498 and .015 . Since the first component, which reflects the covariance of the individuis, is much larger than the second one, it may be conclucied that the subject space is largely onedimensional.

The usefuhess of introducing artificial subjects now becomes clear, as they mark the end-points of the axes. The uniform-scorer and the extreme-scorer demarcate the first axis, and the dominancesubiect and the evaluation-subject take on the extremes of the second axis. The average-subject is located in the middle of the configuration. The random-sibject differs only marginally from the unifom-scorer. The positions of the artificial subjects show that subjects who lie on the first axis of the subject space empha. size the dominance and evaluation axes of the trait space equally strongly. The emphasis increases with increasing scores on this 
Willem A. Van der Kloot and Pieter M. Kroonenberg axis. Subjects on the second axis of the subject space emphasize dominance at the cost of evaluation or vice versa.

The T3-65 core matrix tells the same story (see Table 2). The

Table 2

TS Frontal Planes of Twa Subject Components and Average Frontal Piane

\begin{tabular}{|c|c|c|c|c|c|}
\hline \multicolumn{2}{|c|}{$-1-2-1-1$} & \multicolumn{2}{|c|}{ - } & \multicolumn{2}{|c|}{+2} \\
\hline 71.89 & -2.18 & -9.20 & -2.14 & 31.34 & -2.16 \\
\hline 2.19 & 70.39 & -1.78 & 9.39 & .20 & 39.89 \\
\hline \multicolumn{2}{|c|}{ component 1} & \multicolumn{2}{|c|}{ component 2} & \multicolumn{2}{|r|}{ e plane } \\
\hline
\end{tabular}

diagonal elements of the core plane belonging to the first subject component have equal sizes and the same sign, and indicate therefore that both dominance and evaluation are weighted equally. The diagonal elements of the second subject component also have equal sizes, but opposite signs, indicating that either dominance or evaluation is emphasized. The lavger size of the elements of the first core plane is a direct reflection of the larger eigenvalue of the first subject component as

$$
\sum_{p=1}^{s} \sum_{\eta=1}^{t} c^{2}{ }_{p q r}=\lambda_{r} \quad(\gamma=1,2)
$$

where $\lambda_{r}$ is the $r$ th eigenvalue of the subject space.

Information on the weighting of dominance and evaluation by individual subjects is given by their loadings on the two T3 subject components. In addition, the diagonal elements of each T2 core plane indicate the amount of stretching and shrinking each subject applies to the axes of the common personality space, and the offdiagonal elements indicate the angle under which these axes are 'seen.' It appears that all subjects see these axes as more or less orthogonal because the off-diagonal elements are never really large. Subjects with small and equal diagonal elements in their T2 frontal plane lie on the left hand side of the first axis of the subject space. Subjects with large and equal diagonal elements lie on the right hand side of the first axis, etc. Of the subjects who score most extremely (23, 37, 41 and 55) the core planes are shown in Table 3 , along with an average subject $(47)$, and the average core piane. 
Willem A Van der Kloot and Pieter M. Kroonenberg

Table 3

T2 Frontal Planes of Five Subjects and Average Fronta? Plane

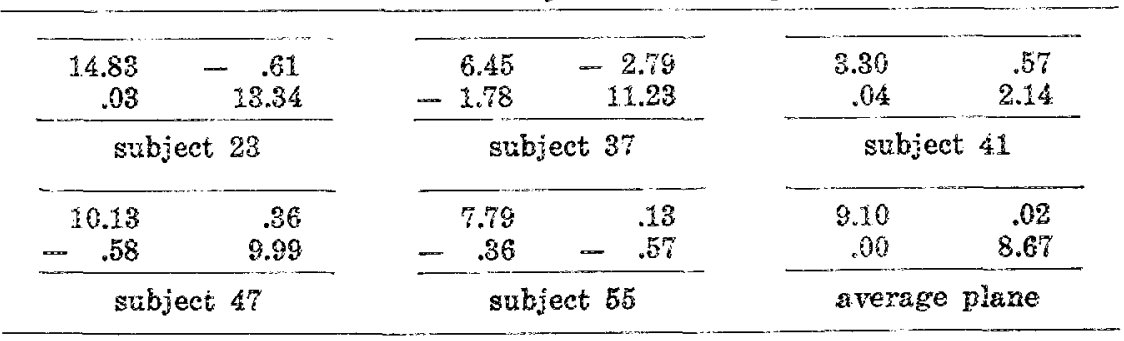

The most important feature of the T3 subject space is thus that most individuals emphasize the dominance and evaluation axes equally but with varying values of the weights. This implies that for most subjects the recovered personality trait configuration (or ITP) is circular, and that some have a larger circle than others. The subjects with large weights (wider circles) have large sums of squares and thus ase most of the ten-point scales. Of secondary importance is that some subjects emphasize either dominance or evaluation. Extreme examples are 55 and 37 , who seem to use either the dominance or the evaluation axis as is confirmed by their T2 core plane in Table 3 .

Cluster analysis and discriminant analysis on the T2 core matrix. The four elements of each frontal plane of the 12 core matrix were taken as scores on four variables, here denoted by $c_{11}$, $c_{12}, c_{21}$, and $c_{22}$. These variables contain information about differences between subjects in their use of the components of the persorality trait space. With the aid of cluster analysis we sought to discover groups of subjects with similar use of these components. The steps to obtain a cluster solution were those recommended in the CLUSTAN manual (Wishart, 1978, p. 10). We applied Ward's method (1963) with the Euclidean distance measure. It was decided to use a five-cluster solution after relocation. To examine the nature of the clusters a discriminant analysis (using BMDPTM, version 2-76; Dixon, 1975) was performed with the clusters as groups and the elements of the $\mathrm{T} 2$ core matrix as variables. Two discriminant functions were found that enabled near perfect classification. Only six points were incorrectly classified by the first discriminant function alone. The scores of the subjects on the two discriminant dimensions were virtually identical to their scores on the T3-65 subject components (canonical correlations between the two sets of coordinates were .977 and .895 ). 
Willem A. Van der Kloot and Pieter M. Kroonenberg

The discriminant weights for $c_{\mathrm{n} 1}$ and $c_{22}$ have the same sign in the first discriminant function, indicating either a stretching or shrinking of the personality trait space, while in the second function $c_{11}$ and $c_{22}$ have opposite signs indicating stretching of one of the axes as well as shrinking the other one. Furthermore the ratio of the eigenvalues of the discriminant functions $(12.4 / 2)$ is comparable to the ratio of the eigenvalues of the two components of the subject space from the T3-59 analysis $(.50, .01)$. This underlines the similarity of both solutions, and shows once more that only the first subject component is really important.

Fit residual ratios. In Figure 3 residual sums of squares are plotted against contributions to the fit for all real and artificial subjects. The heavy line in this figure connects points with the

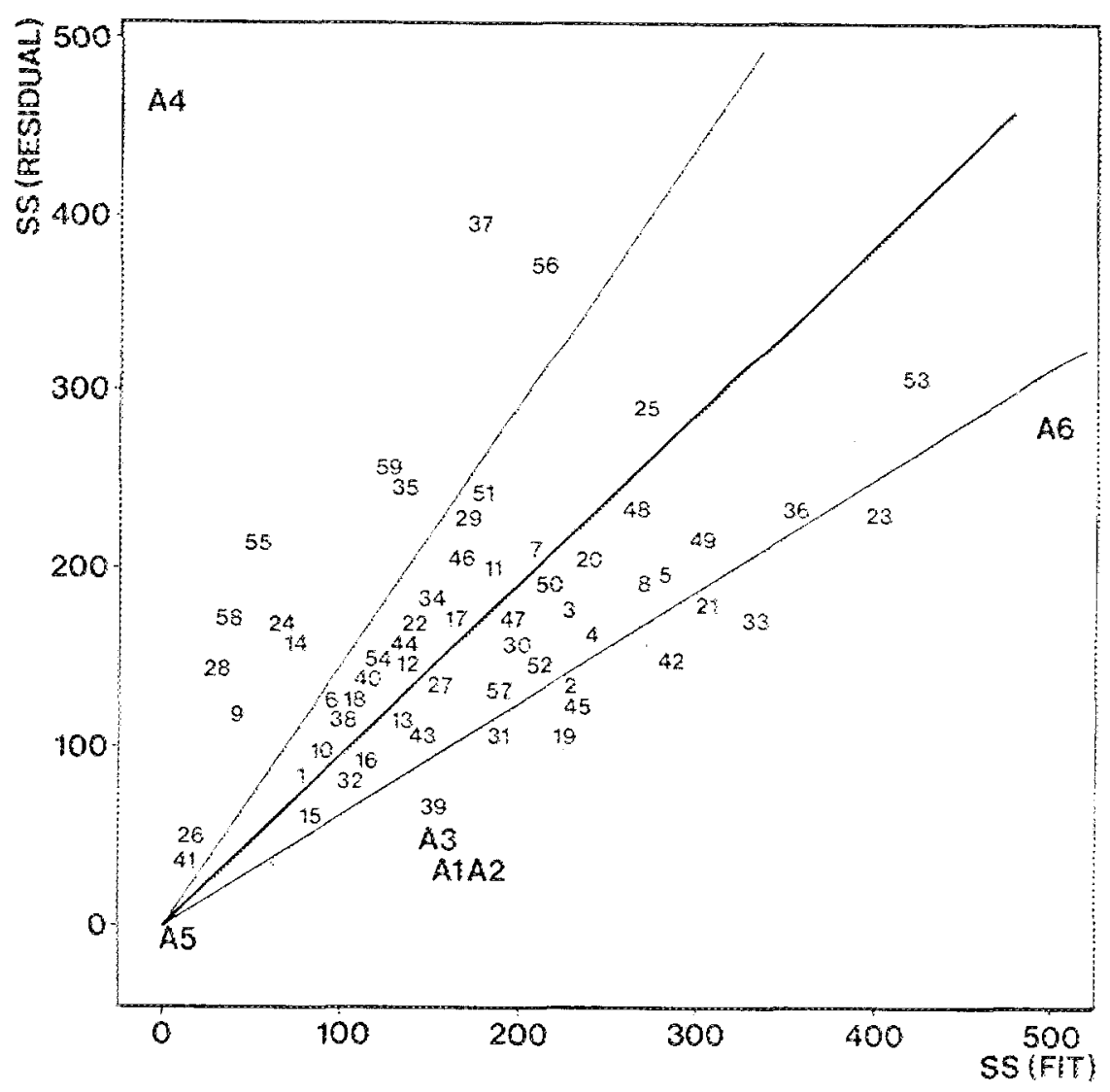

Fig. 3. Plot of each Subject's Residual Sum of Squares versus his or her Fitted Sum of Squares as obtained in the FUCKALS3(65) Analysis. The symbois $A I-A G$ denote the artificial subjects. 
Willem A. Van der Kloot and Pieter M. Kroonenberg

overall fit/residual ratio $(.51 / .49$ for the T3-65 solution). The other two lines connect points with ratios $.61 / .39$ and $.41 / .59$ respectively. These lines serve as a kind of confidence bands for the fit/residuai ratio.

Twelve real sibjects $(9,14,24,26,28,35,37,41,55,56,58$, and 59) have rather small fit/residual ratios, and probably do not meet the assumptions of the model. The "good" points $(2,19,21$, $23,31,33,36,39,42$, and 45) generally have large scores on the first component of the subject space, and thus large overall sums of squares. It is of course not unexpected that subjects with large sums of squares fit better than subjects with small sums of squares.

Also in Figure 3 , the artificial subjects lie on the boundary of the configuration. The random-subject (A4) has practicaly no fit, as it should be. The average-subiect (Al) has roughly the same fit as a real subject (e.g. 17 in the center of the subject space), but due to the averaging procedure, a smaller residual than such an individual. The dominancesubject (A2) and the evaluation-sibject (A3) were created from the average-subject with comparable sums of squares, which explains their position in Figure 3 . The uniformscorer (A5) has fit nor error as his sum of squares is necessarily zero. The extreme-scorer (A6) has understandably a very large sum of squares, and also a better fit/residual ratio $(.64 / .36)$ than the overall one $(.51, .49$ for $13-65)$, which indicates his scoring pattern is admissible in terms of the model. In fact, his private trait space is almost a perfect circle.

\section{Subiects: individual aralysis}

The individual differences described by the core matrix of our $T 2$ and $T 3$ analyses represent those differences only in so far as they remain within the model, i.e., as far as they consist of individual rotations and stretehing or shrinking of the common seale and stimulus spaces. Individual variations that do not conform to the mociel remain undetected and contribute only to the residual sum of squares. In order to explore the possibility of different, individual ITPS 'outside' the T2 and TS model we performed separate two-mode analyses or singular value decompositions on the data of a number of individual subjects with varying fit/residual ratios (including the 12 subjects with the lowest fit/residual ratios).

As was expected, the individual ITPs of the well-fitting subjects corresponded almost completely with the group configurations 
of Figure 1 and 2. However, from the singular values it can be seen that some individual configurations are more ellptical than one would expect from the sizes of the diagonal elements in their T2 core plane. Moreover, the principal components of the individual spaces are not uncommonly at an angle with the principal components of the group space. This means that some subjects have main axes in their ITPs that are not domirance-submission and good-bad, but some other set of polarities (e.g. industriouspassive and aggressive-modest). However, the individual configurations can be generated and interpreted by any set of axes in the trait space. Therefore, it is conceptually more 'parsimonious' to regard the individual axes as linear combinations of the common axes evaluation and dominance.

As we also expected, the correspondence between an indiVidual's separate ITP and the group ITP decreased as the individual's (T3) fit/residual ratio decreased. However, separate analysis did, in general, not dramatically improve a subiect's 'relative fit,' i.e. SS(FIT)/SS(TOTAL), compared with his relative fit in the T3 analysis. Well-fitting subjects of the T3 analysis showed only modest $(.10-15)$ increases in their relative fit, as was to be expected. Separate analyses for 'bad' subjects showed larger increases (up to .35), but the relative fits of their separate analysis never became larger than .63 , which is still noticeably lower than the relative fits of the 'good" subjects (.74-81). In some cases the relative fits were hardy better than 36 , the relative fit of the separate analysis on the random subject. These results suggest that there are no subjects with radically different ITPs. i.e. subjects with a (very) low relative fit in the T3 analysis as welk as a (very) high relative fit in their private solution. In fact low T3 fit tends to go together with low individual fit.

The number and extent of the differences between individual solutions and the T2 or T3 group ITP tend to increase with decreasing fit/residual ratios. The order of the following the types of deviations corresponds roughly with the order in which they oechn when the fit residual ratio decreases.

(a) The circular (or elliptical) shape of the scales and the 11 single stimuli tends to become more angular with decreasing fit residual ratios.

(b) The configurations of some subjects show more or less marked clusterings of the separate scales and stimuli. 
Whlem A. Van der Koot and Pieter M. Kroonenberg

(c) Some configurations exhibit an interchange of the positions on the 'circle" of two or more adjacent scales and 'or stimuli.

(d) A shift of one trait to another region of the 'circle' appears in some configurations.

(e) The stimulus space tends to become increasingly dissimilar to the scale space with decreasing fit/resicual ratios, e.g. some subjects may have a 'reasonable' scale space, but an unrecognizakle stimulus space, and vice versa. This probably indicates inconsistencies or even random errors in the judgments.

The 12 worst-fitting subjects (see Figure 3) have individual configurations that show many or all of the above deviations. Moreover, they differ greatly among themselves. This makes it difficult to assess whether their ITPs are still comparable to the group TTP. The conclusion that their ITPs are not substantively different is suggested by the eigervalues of their individual structures. Three subjects $(9,14,26)$ have eigenvalues that are approximately equal to those (.20 and .17) of the random subject. The remaining nine subjects have structures in which only the first eigenvalue is substantially larger (1.5 times or more) than that of the ravdom subject. The dimensions corresponding to those eigenvalues were (in six cases) associated with the dominance-submission distinction. In one case (37) the main axis was likeable-aggressive, and in two cases it was unclear what the first dimension measured. In the light of these results we conclude that the 12 worst-fitting subjects generated data that are predominantly random error sometimes superimposed on a component of the general ITP.

The above observations are illustrated by the indvidual ITPS depicted in Figure 4. Shown in this figure are the scale configurations of eight subjects $(1,2,9,25,37,41,55$ and 56$)$. Subject 2 is a 'good' subject, with an average total sum of squares. Subjects 1 and 25 are 'averages with a small and a large SS(TOFAL) respectively. Subjects $41,9,55,37$ and 56 are 'bad' subjects with increasing total stms of squares.

\section{DISGUSEION}

From the above results it appears that three-mode principal component analysis is a very useful and appropriate tool for the analysis of our kind of data. The advantage of three-mode analyses is that individual differences are treated within the framework of one mode and that one obtains estimates of the parameters describing such individual differences. 
Willem Av Van der Kloot and Pieter M. Kroonenberg

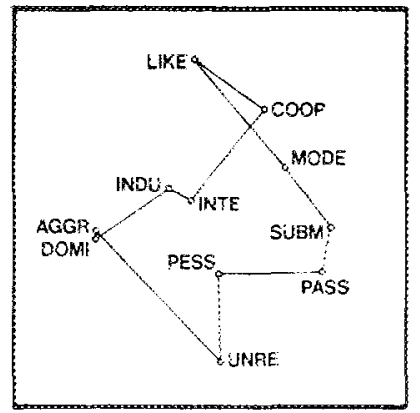

Subject 1

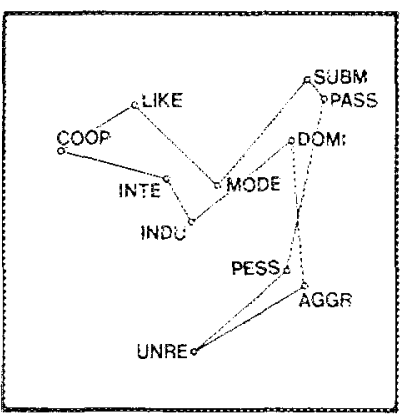

Subject 9

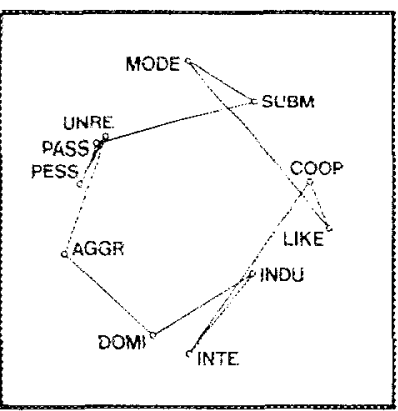

Subject 37

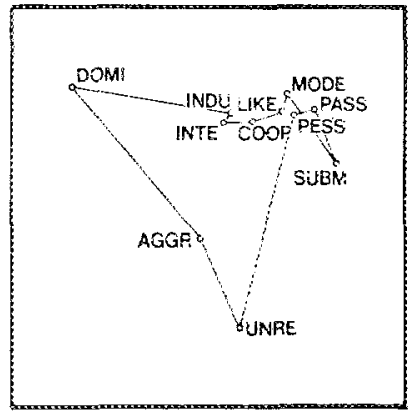

Subject 55

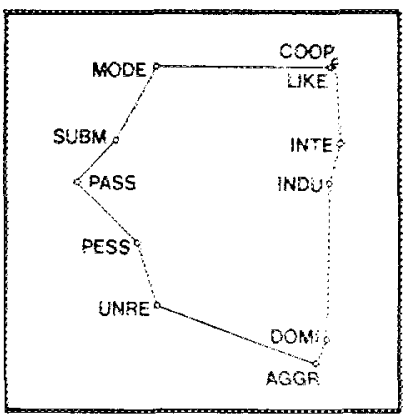

Subject 2

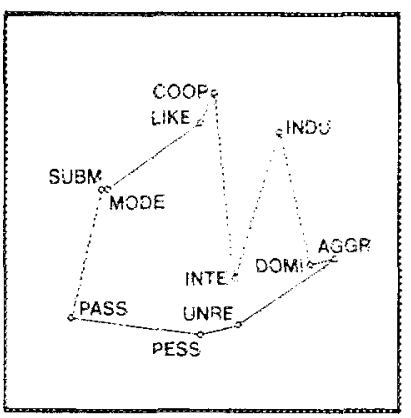

Subject 25

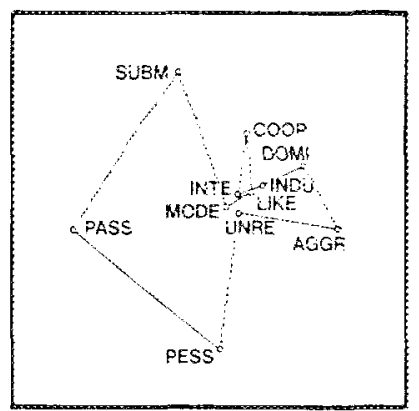

Subject 41

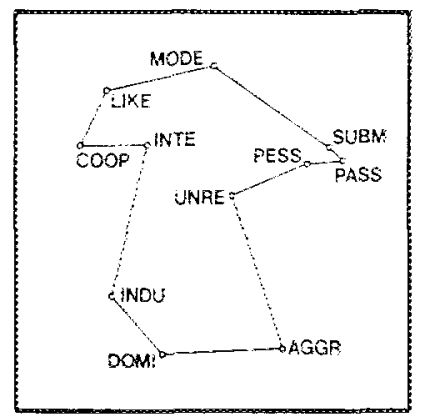

Subject 56

Fig. 4. Eight Exampies of Individual Scale Configurations 
Willem A. Van der Kloot and Pieter $M$. Kroonenberg

A special feature of the Kroonenberg and De Leeuw (1980) version of three-mode principal component analysis is that their treatment of the total sum of squares makes it possible to distinguish between SS(FIT) and SS(RESIDUAL) for each element of each mode (cf. formula [6]). Especially in the case of small weights (saliences) for the group space dimensions it enables the separation of points with a bad fit, as is indicated by a small SS(FIT) and a large SS(RESDDUAL), from points with a small, but welliting configuration, as is indicated by both a small SS(FIT) and a small SS(RESIDUAL). In the first case the data show much variation, but in $a$ way which is not in agreerent with the model; in the second case the relatively little variation is in accordance with the model. Using the residual sums of squares it was possible to select "good" and "bad" subjects, i.e. subjects who conformed to on deviated from the group ITP.

Our final methodological comment concerns the use of "art:ficial"" "conceptual," or "idealized" subjects or "points of view." When using such "subjects" it is especially useful to specify their "ideal" response patterns on the basis of a substantive theory, instead of on the results of the analysis. $\mathrm{By}$ assessing the difference between the real subjects and "ideal" ones, it is possible to accept or reject the models underlying the construction of the latter one and simplify the interpretations of axes.

With regard to the psychological content of our study, our main concern is the extent and nature of individual differences among ITPS. In our presentation of the results we made a distinction between indvidual differences whith and individual differences ontside the three-mode principal component models. Both the T2 and the T3 analyses showed that individual differences allowed by these models were to a very large extent explained by one dimension: the magnitude of the diagonal elements of the subjects' core planes. This means that the largest variation among our subjects consisted of differences in size or area of the individual configurations, i.e. some subjects had large "circles" and others had small "circles." "These results indicate that the subjects had ITPs that were basically similar, and that the inter-subject differences must be attributed to differences in response style: some subjects tended to give more extreme and or more differentiated ratings than others. Such response style differences can be easily detected in the raw data. Some subjects gave only ratings between 4 and 7 , some added an occasional 3 or 8 , and others had data 
Wiliem A. Van der Kloot and Pieter M. Kroonenberg

matrices with many $I$ 's and 10's. Extreme ratings may, in the present case, also be the result of a particular way of drawing inferences. For instance, if a stimulus person was described by trait $A$ and $B$, some subjects always gave 10 ratings on the corresponding $A$ and $B$ scales, whatever the particular combination (e.g. dominant and submissive). Other subjects applied some sort of information-integration process, and gave less extreme ratings (often 5 or 6 ) of the dominance and the submissiveness of this stimulus person. Evidence of these different types of inferential processes was also fond in the subjects' answers on a few open ended questions regarding the way they made their judgments (Vander Kloot and Bakker, Note 1).

Individual differences that are not permitted by the threemode principal component models lead to lower fit/residual ratios for those subjects who do not conform to the model. As separate analyses for a number of individuals showed, lack of fit in the T2 and T3 analyses did not point in the direction of qualitatively different ITPs, but rather indicated partial changes and or chance fluctuations that were superimposed on the common structure. This finding supports the conclusion that our subjects used one common, possibly cuturally shared Implicit Theory of Personalty.

\section{REFERENCE NOTE}

I. Van der Kloot. W. A.s \& Bakker. D. Subjectine response tencencies in impression formation: A content analysis. (Research Note) Cnpublished manuscript. Vakgroep Vethoden er Technieken, Universty of Leiden, The Netheriands, $: 981$.

\section{REFERENCES}

Becker, W. C., \& Krug, R. S. A circumplex madel for social behavior in children. Child Development, 1964, 35,371-396.

Berjamin, L. S. Structural analysis of social behavior. Pencholagical Revieu, 1974. $81,392-425$.

Bruner, J. S., \& Tagiur, R. The perception of people. In G. Lindzey (Ed.), Handbook of social psychology (Vol, 2). Cambriage, Mass.: AddisonWesley, 1954.

Bryson, J. B. Factor analysis of impression formation processes. Journat of Personality and Social Psychology, 1974, 30, 134-143.

Clff, $\mathbb{N}$. The "idealized individual" interpretation of individual differences in multi-dimensional scaling. Pevchometrikn, 1968, s2, 225-232.

Cronbach, L. J. Processes affecting scores on "understanding of others" and "assumed similarity". Psychological Bulletin, 1955, 52, 157-193.

Dixon. W. J. (Ed.) BMDP Biomedical Computer Frograme, Berkeley, California: Lniversity of Calfomia Press, 2975. 
Willem A. Van der Kioot and Pieter M. Kroonenberg

Freedman, M. B., Leary. T. F, Ossorio, A. G., \& Coffey, H. S. The interpersonal dimensions of personality. Journal of Personalit $, 1951,20,153-$ 161.

Hays, W. L. An approach to the study of irait implications and trait simiIarity. In R. Tagiur, \& L. Petrullo (Eds.), Person perception and inter. personal bohaviom. Stanford: Stanfor University Press, 1958.

Jones, E. E. Autboritarianism as a deteminant of first-impression formation. Journat of Personality. 1954,23, $107-127$.

Kim, M. P., \& Rosenberg, S. Comparisor of two structural modeis of implieit personality theory. Journal of Personality and Sociti Psychologu, 1980 , $88,375-389$.

Kroonenbere, P. M., \& De Leeww, J. TUCKALS2: A principal component. analysis of three-mode data (Repont 001-77). Leiden, The Netherlands: Department of Data Theory, University of Leiden, $197 \%$.

Kroonenberg, P.M., \& De Leeuw. I. Principal component analysis of threemodie data by means of aitemating least squares algorithms, Psychometrita, $1980,45,69-97$.

Lay, C. H., \& Jackson, $D . N$. Analysis of the generality of trait-inferential relationships, Joumal of Personality and Social Psychology, 1969, i2, i2-21.

Leary, T. F. Interpersonal diagnosis of personality. New York: Ronald, 1957.

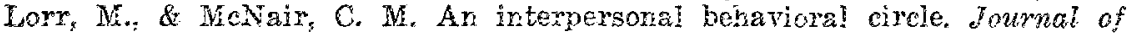
Abnomal and Social Psuchology. 1963,67,68-75.

Messick, S. \& Kogun, N. Personality inconsistencies in ixdgements: Dimen. sions of role construets. Maltivariate Behanoral Research, 1966, 1.65-176.

Pederson, $D . M$. The measurement of individual ufferenees in pereeived pexsonality-trait relationships and their relation to ceriain determinants. Joumal of Socin Psychology, 1955, 65, 233-258.

Rinn. J. L. Structure of phenomenal domains. Psychological Review, 1965, 7R, $455-466$.

Rosenberg, $S$. New anproaches to the analysis cf personal constructs in person perception. In J. K. Cole \& A. W. Landsfield (Eds), Igre Nebraska symposium on motivation. Lincoln, Nebrasha: University of Nebraska Press. 2077.

Roserberg, S., Nelson, C, \& Vyekananthan, P. S. A multidimensional approach to the structure of personality impressions. Joumal of Personaity and Socia: Peychology, 1968, $9,283-294$.

Schneider, D. I. Implict personality theory: A review, Psychological Bulletin, $1973,79,294-308$.

Sheman, R.C. Individual differences in pereeived trait relationships as a function of dimensional salience. Mhultionriate Behavioral Research, 1972 . $7,109-229$.

Tucker, L. R. Some mathematical notes on three-mode factor analysis. Psychometritin, $1966,21,279-311$.

Tucker, L. R. Reiations between mulicimengional sealing and three-moce

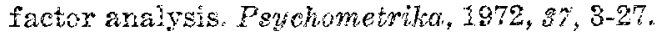

Tucker, L. R., \& Messick, S. An indvidual difference model for multidimensionai sealng. Peychometriko, $1963,28,383-367$.

Van de Geer, J. P. Introduction to multivariate analusis for the social sciences. San Francisco: Freemar, 197.

Van der Bloot, W. A. A cognitive struetwe approach to person perception: Five studies on the implicit theory of personalizy. Leiden, The Netherlands: Author, 1975 .

Van der Kloot, W. A., \& Var den Boogaard. T. Weighte of information in impression formation (Report MT. 001-78). Leiden, The Netheriands: Department of Psychology, University of Leicen, 1978.

Walters, $H$. \& Jackson, D. Group and individual regularities in trait inference: A multi-dimensional scaling analysis. Multivariate Behavioral Research, $1966,1,545-163$. 
Willem A. Van der Kloot and Pieter M. Kroonenberg

Ward, J.F. Hierarchical grouping to optimize an objective function. Journal of the American Statistical Association, $3963,58,236-244$.

Wiggins, J. S. A psychological taxonomy of trait-descriptive terms: The intempersonal domain. Joumal of Personality and Social Psychology, $1979,8 \%, 395-412$.

Wiggins, J. S. Steiger, J. I., \& Gaelick, L. Evaluating cireimplexty in personality data. Wutivariate Behavioral Research, $2053,16,263-289$.

Wiggins, N., \& Blackburn, M. C. Implicit treories of personaty: An indiridual differences approach. Wuitivariate Behavioral Research. 1970, 1 , $265-285$.

Wggins, N., Hoffman, P. I. \& Taber, T. Types of judges and cue utilization in tudgmente of intelligence. Toumal of Personality and Social Psychotogy, $1969,19,52-59$.

Wishart, D. CLUSTAN user manual (3rd Ed.). Edinburgh: Progran Library Unit, Edinburgh University, 1978 .

Wishrer, J. Information redundancy, inconsistency, and novelty and their role in impression formation. Journal of Experimental Social Psichoiogy, $1960,6,111-127$.

Zanna, M. P., \& Hamilton. D. L. Attribute dimensions and pattems of trait inferences. Psychoromic Science, 1972, 27, 353-354. 